cancer related lymphoedema. However, over time it became apparent that the condition for many of these patients had stabilised and they were living with chronic lymphoedema. This posed a dilemma for the hospice as to whether hospice funds should continue to be used to treat this category of 'non-palliative' patients. The hospice undertook a detailed activity analysis of the lymphoedema service and presented this to the NHS commissioning managers. This led to the hospice working with one PCT area to establish an NHS community based non-palliative lymphoedema service. A further, newly formed CCG decided to take a different route to offer a non-palliative service under an Any Qualified provider (AQP) contract.

Aim To establish a non-palliative lymphoedema service under an AQP contract as an extension to the existing experienced hospice lymphoedema service.

Approach used

- Hospice managers attended AQP stakeholder meeting.

- Submission of tender documentation.

- Acceptance of contract.

- Redesign of current service to accommodate new contract for wider geographical area.

- Recruitment, training of additional staff.

- Set up of data collection to meet requirements of contract.

- Setting up good working relationships with the other 3 commissioned AQP providers.

Outcomes

- Patients who are classed as non-palliative have the benefit of an excellent well established palliative lymphoedema service.

Application to hospice practice.

- The hospice now receives full funding for non-palliative patients.

- The hospice lymphoedema team are able to access additional training and gain knowledge of different conditions, this ultimately extends the range of skills and experience that they have to hand to support palliative patients.

- Non-palliative patients more prepared to accept hospice care should they have further disease recurrence or their condition deteriorates.

- Opportunity for hospice managers to gain experience in AQP commissioning process.

\section{ONE STEP BEYOND: WORKING IN PARTNERSHIP WITH CCGS TO EXTEND ACCESS TO BEREAVEMENT SERVICES}

${ }^{1} J$ une Patel, 'Sarah Popplestone Helm, ${ }^{2}$ Debbie Westwood; 'St Richards Hospice, Worcester, UK, ${ }^{2}$ CAREWISE Worcestershire County Council, ${ }^{3}$ AGE UK Worcester, ${ }^{4}$ South Worcestershire Clinical Commissioning Group

\subsection{6/bmjspcare-2013-000591.18}

Aims The service aims to - Provide access to bereavement support for all bereaved people irrespective of age, culture disability and gender - Assess bereavement need and signpost people pre and post bereavement to bereavement services within their locality - Extend access to advice 24/7 supported by the development of a bespoke bereavement website www.bereavementsupportworcestershire.org.uk telephone help-line service, Counselling and Social Work support - Raise professional awareness about grief by providing bespoke education and training - Raise awareness of the bereavement process and coping strategies of bereaved individuals and families by education and group support.
Approach A formal tendering process resulted in securing a three year NHS contract. A multi-agency project group was formed, including a service user, to develop the operational service model in accordance with the NHS service specification. Outcomes Widening access of bereavement support to adults and children experiencing sudden death from suicide, RTAs' and unexpected deaths - Multi-agency engagement in shaping operational model - Rapid access to social work and counselling social for the bereaved following self-referral and referrals from Primary Care - Positive evaluations from services users and GPs' - Service user involvement in the formal media launch with South Worcestershire CCG - 193 professionals have attended education programme and positively evaluated - 7401 hits to access information on bereavement website.

\section{Conference Papers 5}

\section{RECOGNISING DYSPHAGIA RISK AND COMPROMISED NUTRITION IN PATIENTS WITH ADVANCED CHRONIC OBSTRUCTIVE PULMONARY DISEASE (COPD): EXPLORING THE ROLE OF SPEECH AND LANGUAGE THERAPY AND DIETETICS}

Sarah Harvey, Lianne Gordon; St Joseph's Hospice, London, United Kingdom

\subsection{6/bmjspcare-2013-000591.19}

Background Patients with advanced COPD have an increased risk of dysphagia ${ }_{1}$ and malnutrition ${ }_{2}$. These symptoms are frequently inter-related and can have a detrimental impact on patients' nutritional wellbeing, frequency of exacerbations and quality of life. Speech and Language Therapists (SLT) and Dietitians possess expertise in the diagnosis and management of dysphagia and malnutrition however their role supporting palliative patients with advanced COPD is not well established.

Aim To explore the role of SLT and Dietitian in the screening, diagnosis and management of dysphagia and malnutrition in patients with advanced COPD.

To raise awareness of swallowing and nutrition difficulties in advanced COPD amongst patients, carers and palliative care professionals.

Approach Used All hospice patients with a diagnosis of advanced COPD were screened for dysphagia and malnutrition by the SLT and Dietitian over a one month period. Anecdotal qualitative information was gathered on the impact of COPD on aspects of everyday life relating to feeding including shopping, food preparation, fatigue, appetite, ability to eat a meal.

Outcomes The majority of hospice patients with advanced COPD presented with a degree of oropharyngeal dysphagia and malnutrition (weight loss and fatigue). Patients were unaware that eating and drinking was affected by COPD, believing that swallowing difficulties and loss of appetite were things they would 'have to put up with'. Patients were unaware that help was available and had no understanding of the role of SLT and Dietitian in supporting their symptom management.

Application to hospice practice SLT and Dietitian appear to have important roles in raising awareness and supporting best management of dysphagia and malnutrition in patients with advanced COPD, to optimise their feeding and nutritional wellbeing.

Hospices caring for patients with advanced COPD should proactively screen for dysphagia and malnutrition to ensure these symptoms are recognised and appropriately managed. 